\title{
Hyperbolic triangular buildings without periodic planes of genus two
}

\author{
Riikka Kangaslampi, Alina Vdovina
}

October 20, 2015

\begin{abstract}
We study surface subgroups of groups acting simply transitively on vertex sets of certain hyperbolic triangular buildings. The study is motivated by Gromov's famous surface subgroup question: Does every one-ended hyperbolic group contain a subgroup which is isomorphic to the fundamental group of a closed surface of genus at least 2? In 9] and [3] the authors constructed and classified all groups acting simply transitively on the vertices of hyperbolic triangular buildings of the smallest non-trivial thickness. These groups gave the first examples of cocompact lattices acting simply transitively on vertices of hyperbolic triangular Kac-Moody buildings that are not right-angled. Here we study surface subgroups of the 23 torsion free groups obtained in 9. With the help of computer searches we show, that in most of the cases there are no periodic apartments invariant under the action of a genus two surface. The existence of such an action implies the existence of a surface subgroup, but it is not known, whether the existence of a surface subgroup implies the existence of a periodic apartment. These groups are the first candidates for groups that have no surface subgroups arising from periodic apartments.
\end{abstract}

\section{Introduction}

In [9] the authors classified all torsion-free groups acting simply transitively on the vertices of hyperbolic triangular buildings of the smallest non-trivial thickness. They constructed the groups with the polygonal presentation method introduced in 13. As a result, they obtain 23 non-isomorphic groups, each defined by 15 generators $x_{1}, x_{2}, \ldots, x_{15}$ and 15 cyclic relations, each of them of the form $x_{i} x_{j} x_{k}=1$, where not all the indices are the same. The underlying hyperbolic building is the universal cover of the polyhedron glued together from 15 geodesic triangles with angles $\pi / 4$ and with the letters from the relations written on the boundary. In constructing the polyhedron the sides of the triangles with the same labels are glued together, respecting the orientation. For example, the presentations $T_{1}, T_{3}, T_{9}$ and $T_{21}$ obtained in [9] are given in Table 1. These will be used later as examples.

Thus these sets of 15 triangles, with angles $\pi / 4$, words specified in [9] written at the boundary and glued together respecting orientation, all give a polyhedron that has one vertex and the smallest generalised quadrangle as the link. The universal cover of this polyhedron is a hyperbolic triangular building [1, and 


\begin{tabular}{|l|l|l|l|}
\hline$T_{1}$ & $T_{3}$ & $T_{9}$ & $T_{21}$ \\
\hline$\left(x_{1}, x_{1}, x_{10}\right)$ & $\left(x_{1}, x_{1}, x_{10}\right)$ & $\left(x_{1}, x_{1}, x_{10}\right)$ & $\left(x_{1}, x_{5}, x_{2}\right)$ \\
$\left(x_{1}, x_{15}, x_{2}\right)$ & $\left(x_{1}, x_{15}, x_{2}\right)$ & $\left(x_{1}, x_{15}, x_{2}\right)$ & $\left(x_{4}, x_{13}, x_{11}\right)$ \\
$\left(x_{2}, x_{11}, x_{9}\right)$ & $\left(x_{2}, x_{11}, x_{3}\right)$ & $\left(x_{2}, x_{11}, x_{4}\right)$ & $\left(x_{1}, x_{6}, x_{4}\right)$ \\
$\left(x_{2}, x_{14}, x_{3}\right)$ & $\left(x_{2}, x_{14}, x_{5}\right)$ & $\left(x_{2}, x_{14}, x_{6}\right)$ & $\left(x_{5}, x_{9}, x_{10}\right)$ \\
$\left(x_{3}, x_{7}, x_{4}\right)$ & $\left(x_{3}, x_{7}, x_{4}\right)$ & $\left(x_{3}, x_{5}, x_{9}\right)$ & $\left(x_{1}, x_{3}, x_{13}\right)$ \\
$\left(x_{3}, x_{15}, x_{13}\right)$ & $\left(x_{3}, x_{15}, x_{8}\right)$ & $\left(x_{3}, x_{8}, x_{7}\right)$ & $\left(x_{5}, x_{13}, x_{9}\right)$ \\
$\left(x_{4}, x_{8}, x_{6}\right)$ & $\left(x_{4}, x_{8}, x_{9}\right)$ & $\left(x_{3}, x_{10}, x_{13}\right)$ & $\left(x_{2}, x_{7}, x_{10}\right)$ \\
$\left(x_{4}, x_{12}, x_{11}\right)$ & $\left(x_{4}, x_{12}, x_{12}\right)$ & $\left(x_{4}, x_{8}, x_{5}\right)$ & $\left(x_{6}, x_{9}, x_{8}\right)$ \\
$\left(x_{5}, x_{5}, x_{8}\right)$ & $\left(x_{5}, x_{9}, x_{6}\right)$ & $\left(x_{4}, x_{14}, x_{14}\right)$ & $\left(x_{2}, x_{12}, x_{15}\right)$ \\
$\left(x_{5}, x_{10}, x_{12}\right)$ & $\left(x_{5}, x_{13}, x_{13}\right)$ & $\left(x_{5}, x_{10}, x_{12}\right)$ & $\left(x_{6}, x_{11}, x_{10}\right)$ \\
$\left(x_{6}, x_{6}, x_{14}\right)$ & $\left(x_{6}, x_{8}, x_{11}\right)$ & $\left(x_{6}, x_{7}, x_{12}\right)$ & $\left(x_{3}, x_{11}, x_{14}\right)$ \\
$\left(x_{7}, x_{7}, x_{12}\right)$ & $\left(x_{6}, x_{10}, x_{13}\right)$ & $\left(x_{6}, x_{15}, x_{9}\right)$ & $\left(x_{7}, x_{8}, x_{15}\right)$ \\
$\left(x_{8}, x_{13}, x_{9}\right)$ & $\left(x_{7}, x_{9}, x_{14}\right)$ & $\left(x_{7}, x_{8}, x_{11}\right)$ & $\left(x_{3}, x_{14}, x_{8}\right)$ \\
$\left(x_{9}, x_{14}, x_{15}\right)$ & $\left(x_{7}, x_{10}, x_{12}\right)$ & $\left(x_{9}, x_{15}, x_{13}\right)$ & $\left(x_{7}, x_{14}, x_{12}\right)$ \\
$\left(x_{10}, x_{13}, x_{11}\right)$ & $\left(x_{11}, x_{15}, x_{14}\right)$ & $\left(x_{11}, x_{12}, x_{13}\right)$ & $\left(x_{4}, x_{12}, x_{15}\right)$ \\
\hline
\end{tabular}

Table 1: Presentations $T_{1}, T_{3}, T_{9}$ and $T_{21}$ from [9].

the group with 15 generators $x_{1}, x_{2}, \ldots, x_{15}$ and the 15 words from the boundaries of the triangles as relations, acts on the building cocompactly and simply transitively.

These groups are the first examples of cocompact lattices acting simply transitively on vertices of hyperbolic triangular Kac-Moody buildings that are not right-angled. For a general introduction to the theory of hyperbolic buildings and their lattices, see the survey $[12$ by A. Thomas.

Here we study the 23 groups further, motivated by Gromov's famous surface subgroup question: Does every one-ended hyperbolic group contain a subgroup which is isomorphic to the fundamental group of a closed surface of genus at least 2? Recall, that a group $G$ is a surface group if $G=\pi_{1}(\mathcal{F})$, where $\mathcal{F}$ is a closed surface. If in addition $\mathcal{F}$ is finite, then $\pi_{1}(\mathcal{F})$ has one of the following forms (see [11])

(i) $G=\left\langle a_{1}, b_{1}, \ldots, a_{n}, b_{n} \mid a_{1} b_{1} a_{1}^{-1} b_{1}^{-1} \cdots a_{n} b_{n} a_{n}^{-1} b_{n}^{-1}\right\rangle$, when $\mathcal{F}$ is orientable and of genus $n$,

(ii) $G=\left\langle a_{1}, \ldots, a_{n} \mid a_{1}^{2} a_{2}^{2} \cdots a_{n}^{2}\right\rangle$, when $\mathcal{F}$ is non-orientable and of genus $n$.

Gromov's question remains open, but there are many classes of hyperbolic groups, for which the answer is positive. For example, see [4 for surface subgroups of right-angled Artin groups or [2, where Calegari and Walker show, that a random group contains many quasiconvex surface subgroups. Existence of surface subgroups in right-angled hyperbolic buildings was shown in [5], and in hyperbolic buildings with 4-gonal apartments in [14]. Existence of surface subgroups in fundamental groups of higher-dimensional complexes is discussed, for example, in [6].

We are especially interested in periodic apartments, invariant under an action of a surface group, since such an action implies an existence of a surface subgroup. For periodic apartments in Euclidean buildings using dynamics, see [1]. In [14, periodic apartments were shown to exist in some hyperbolic buildings. 
It is not known, whether the existence of a surface subgroup implies the existence of a periodic apartment. In this paper we obtain first candidates for groups not having periodic apartments. We show, that one cannot find an apartment invariant under a genus two surface group action in most of the considered 23 groups.

\section{Periodic apartments of genus 2}

Theorem 2.1. There are hyperbolic triangular buildings admitting simply-transitive torsion free action and having the smallest generalised quadrangle as the link at each vertex that do no have any apartments invariant under genus 2 orientable surface group action.

Proof. Let us assume, that there exists an action of genus 2 surface on an apartment of the building. Let's consider the triangulation of the surface induced by this action and take the dual graph of this triangulation. It has a vertex for each triangle of which the surface is glued together, and edge between two vertices, if the corresponding triangles are adjacent. Thus the dual graph is 3-valent. Since the triangles have angles $\pi / 4$, eight of them must meet at any vertex of the surface. It means that in the dual graph there are cycles of length eight, or, in other words, we can think about the surface also as being glued together from octagons (see Figure 1). Note that the same triangle can appear more than once in an octagon, so we should in fact talk about closed walks of length 8 , but, for simplicity, let us call them 8-cycles. Since the edges in the dual graph each correspond to an edge in the triangulation, the labelling of the edges of the triangles with $x_{1}, \ldots, x_{15}$ corresponds to a colouring of the edges in the dual graph.

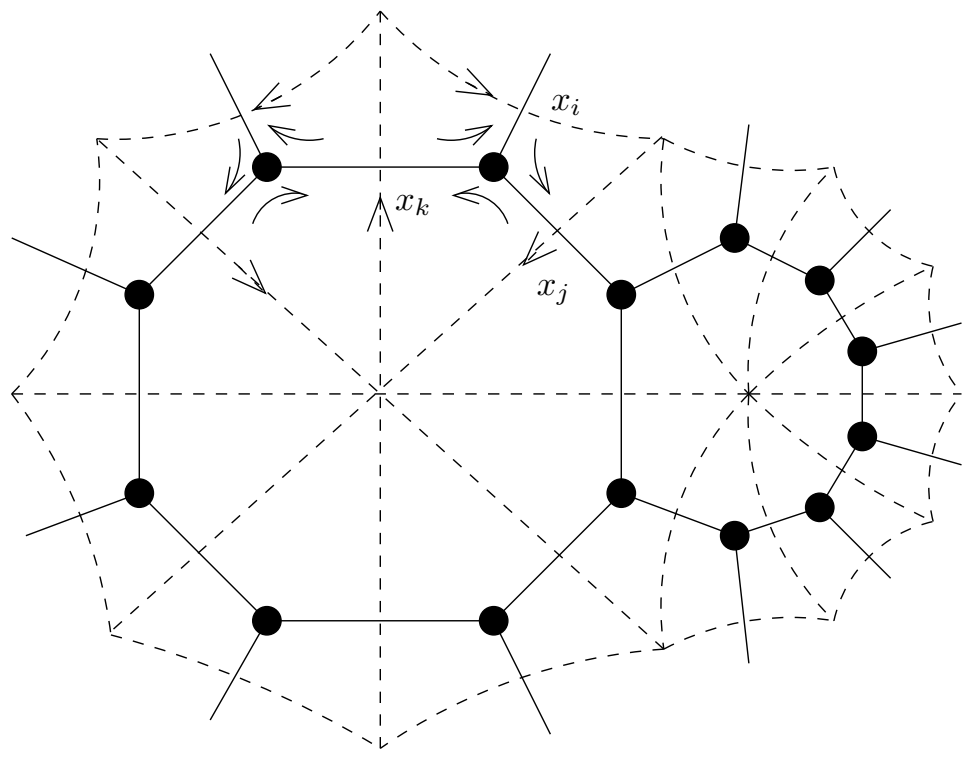

Figure 1: The edges of the dual graph get their labels from the sides of the triangles. 
From Euler's formula $V-E+F=2-2 g$ we can now deduce the number of triangles in a surface of genus 2: The surface is tessellated by regular octagons. Three of them meet at each vertex, since the dual graph is 3-valent, and each edge is shared by two octagons. Thus if we denote the number of octagons by $F$, the number of edges is $4 F$ and the number of vertices is $8 F / 3$, and we get

$$
F=6 g-6 .
$$

So, for a genus 2 surface we need 6 octagons, and thus 16 triangles. The dual graph therefore has 16 vertices and 24 edges.

Since the triangles are oriented, they induce an orientation to the vertices of the dual graph (Figure 11). Two adjacent triangles have different orientation, and so also two adjacent vertices in the dual graph have different orientation. Thus the dual graph must be bipartite. Denote, that the vertices of the 8-cycles at the boundaries of the octagons have alternating orientations.

It is possible that some of the triangles forming the surface are glued together from two sides. This means that there is a double edge between two vertices in the dual graph. However, let us first consider the dual graphs with no double edges. Such graphs have girth at least 3 .

\subsection{Dual graphs without double edges}

From Gordon Royle's list of cubic graphs [7] we see that there exist 4060 cubic graphs with 16 vertices, 24 edges and girth 3 or more. We generate all of these using nauty [10]. Only 38 of them are bipartite. By a computer programme written in Fortran we check the existence of six 8-cycles in these graphs with a depth first algorithm as follows.

We pick one of the vertices as the starting point for the search. There has to be three octagons through this vertex. So we can pick any of the adjacent ones to be another vertex in the first octagon. Then we proceed along the graph, not visiting the same vertex twice, until we arrive to the eighth vertex. If this the one we started from, we have an octagon. If not, we go back one step at the time, trying all the other possible ways to proceed from the previous vertex. We keep track of everything we have tried. When one octagon is found, we proceed searching for another 5. Each edge in the graph must be used in two different octagons, once to each direction.

Only the graphs that have numbers 3345, 3538, 3621, 4002 and 4060 when all the 4060 are generated with nauty are bipartite and have 6 cycles of length 8 in them, and thus only these five graphs fulfill the above conditions for a dual graph of a surface of genus 2. Let us call these graphs $G_{3345}^{0}, G_{3538}^{0}, G_{3621}^{0}$, $G_{4002}^{0}$ and $G_{4060}^{0}$. Note, that the set of six octagons is not unique in any of these five graphs: in the graph $G_{3345}^{0}$ there are 8 ways to pick a set of six octagons with the desired properties. In the graph $G_{3538}^{0}$ there are 2 ways to pick the set, in $G_{3621}^{0} 6$ ways, in $G_{4002}^{0} 48$ ways and in the graph $G_{4060}^{0} 18$ ways to pick the set of octagons.

One set of six octagons in the $G_{3345}^{0}$ (Figure 2) is given in Table 2 as a list of vertices. The cycles induce an orientation to the vertices, i.e. following the arrows in Figure 2 we obtain the octagons. This orientation has to be reversed in every other vertex to obtain the orientation of the triangles centered at these vertices of the dual graph. Obviously, all the cycles can also be taken to the opposite orientation, resulting to the opposite orientation at all vertices. 


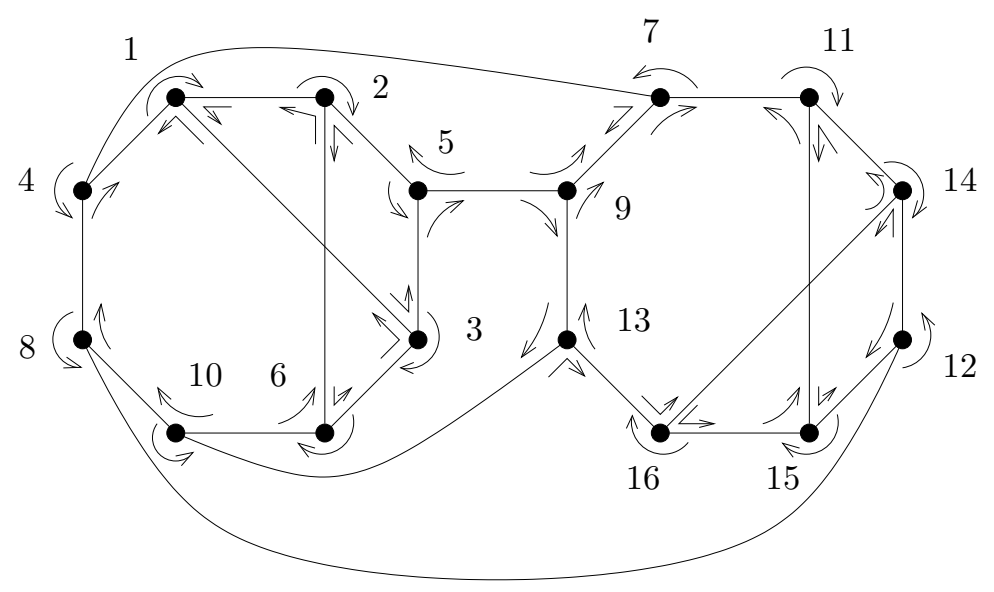

Figure 2: Graph $G_{3345}^{0}$ with orientations that give six 8-cycles.

\begin{tabular}{|l|l|}
\hline Graph $G_{3345}^{0}$ & Graph $G_{3538}^{0}$ \\
\hline$(1,2,5,3,6,10,8,4)$ & $(1,2,5,3,7,10,6,4)$ \\
$(1,3,5,9,13,10,6,2)$ & $(1,3,5,9,8,4,6,2)$ \\
$(1,4,7,9,5,2,6,3)$ & $(1,4,8,12,15,11,7,3)$ \\
$(4,8,12,14,16,15,11,7)$ & $(2,6,10,14,16,13,9,5)$ \\
$(7,11,14,12,15,16,13,9)$ & $(7,11,13,16,15,12,14,10)$ \\
$(8,10,13,16,14,11,15,12)$ & $(8,9,13,11,15,16,14,12)$ \\
\hline
\end{tabular}

Table 2: Examples of a set of six octagons in the graphs $G_{3345}^{0}$ and $G_{3538}^{0}$.

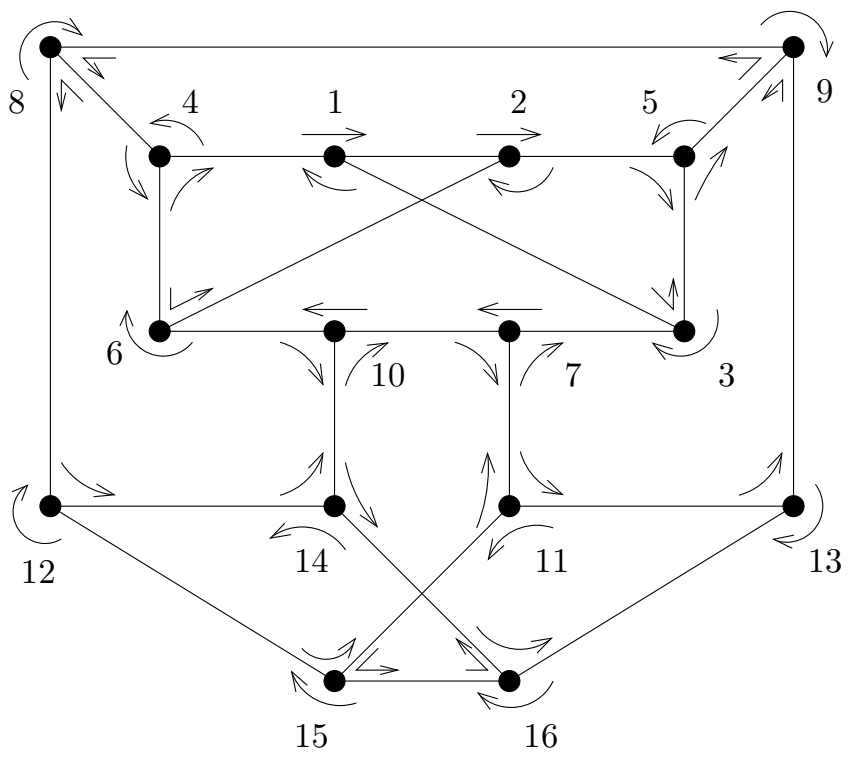

Figure 3: Graph $G_{3538}^{0}$ with orientations that give six 8-cycles. 
A set of cycles of length 8 in the graph 3538 (Figure 3) are presented in Table 2 and sets of cycles in the graphs $G_{3621}^{0}$ (Figure 4), $G_{4002}^{0}$ (Figure 5) and $G_{4060}^{0}$ (Figure 6) are given in Table 3. Also in these figures is denoted the orientation of the vertices that corresponds to the octagons, and the orientations of the triangles are obtained reversing the orientation at every other vertex.

\begin{tabular}{|l|l|l|}
\hline Graph $G_{3621}^{0}$ & Graph $G_{4002}^{0}$ & Graph 4060 \\
\hline$(1,2,5,9,14,10,6,4)$ & $(1,2,6,11,15,13,7,3)$ & $(1,2,6,14,9,12,8,3)$ \\
$(1,3,7,11,8,4,6,2)$ & $(1,3,5,10,15,11,8,4)$ & $(1,3,7,11,5,12,9,4)$ \\
$(1,4,8,13,15,9,5,3)$ & $(1,4,9,12,16,10,5,2)$ & $(1,4,10,15,8,12,5,2)$ \\
$(2,6,10,16,12,7,3,5)$, & $(2,5,3,7,14,16,12,6)$ & $(2,5,11,16,15,10,13,6)$ \\
$(7,12,15,13,16,10,14,11)$ & $(4,8,13,15,10,16,14,9)$ & $(3,8,15,16,14,6,13,7)$ \\
$(8,11,14,9,15,12,16,13)$ & $(6,12,9,14,7,13,8,11)$ & $(4,9,14,16,11,7,13,10)$ \\
\hline
\end{tabular}

Table 3: Examples of a set of 6 octagons in the graphs $G_{3621}^{0}, G_{4002}^{0}$ and $G_{4060}^{0}$.

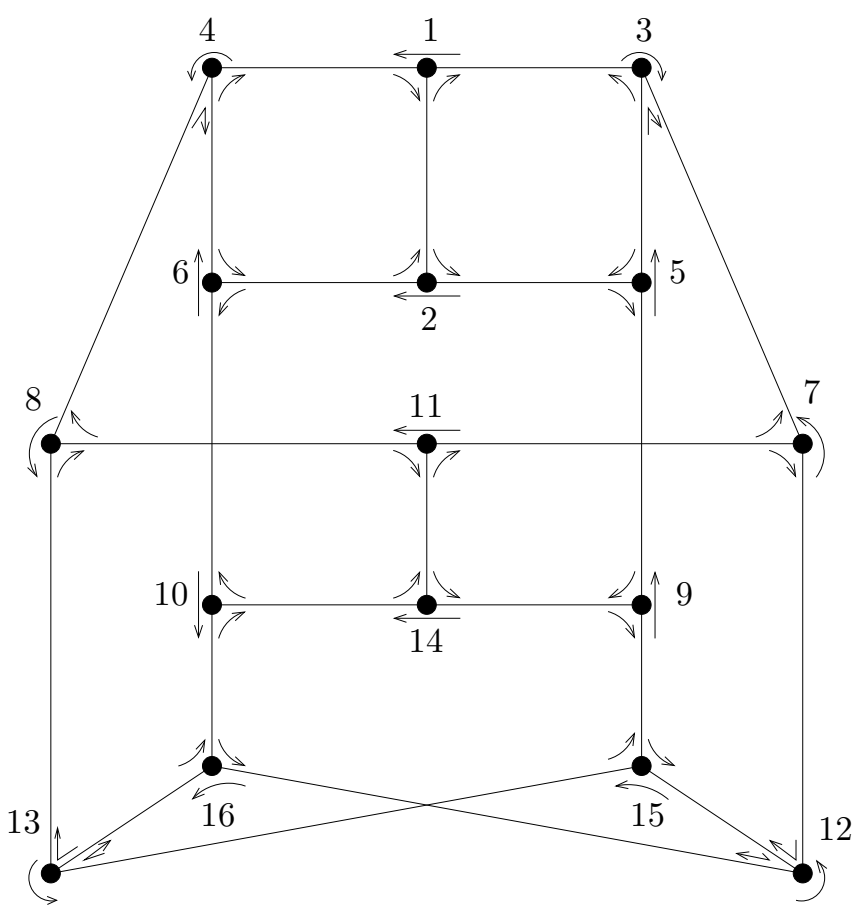

Figure 4: Graph $G_{3621}^{0}$ with orientations that give six 8-cycles.

With the help of a another computer programme we then go through all the 23 sets of triangles for each of these 5 graphs, and for each graph for all the sets of six octagons in it. We look for for a labelling of the edges of the dual graphs in such a way that around each vertex the labelling of the three edges adjacent to it corresponds to a labelling of one of the triangles, respecting orientation. Adjacent vertices cannot get their labelling from the same triangle, unless the triangle has the same label for two edges.

Let us take for example the graph $G_{3621}^{0}$ with the 8-cycles specified in Table 3 and search for colouring by $T_{21}$. There are $15 \times 3$ ways to choose the triangle 


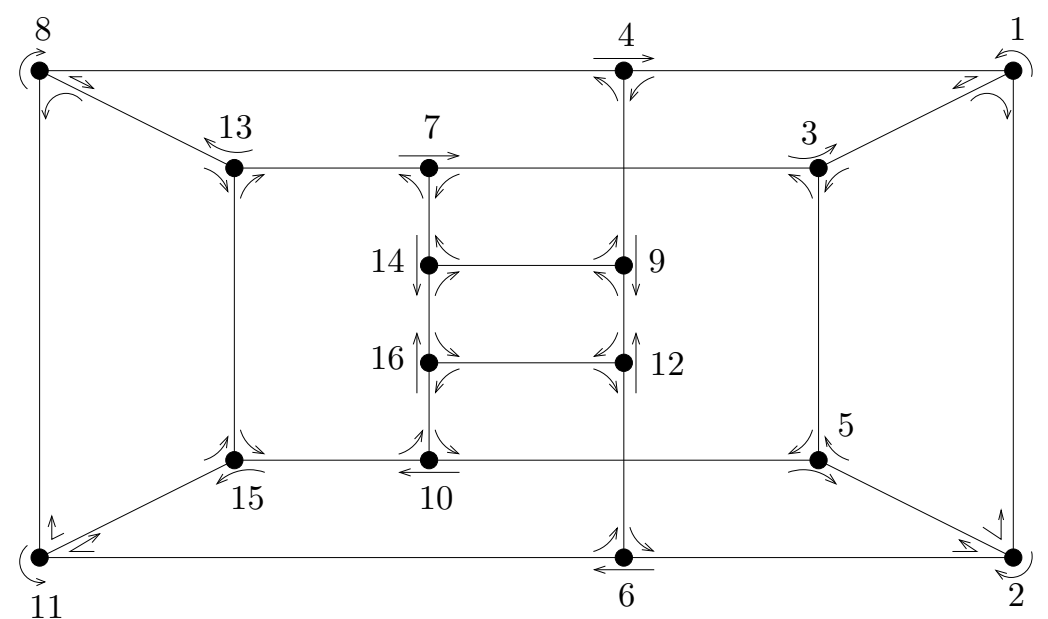

Figure 5: Graph $G_{4002}^{0}$ with orientations that give six 8-cycles.

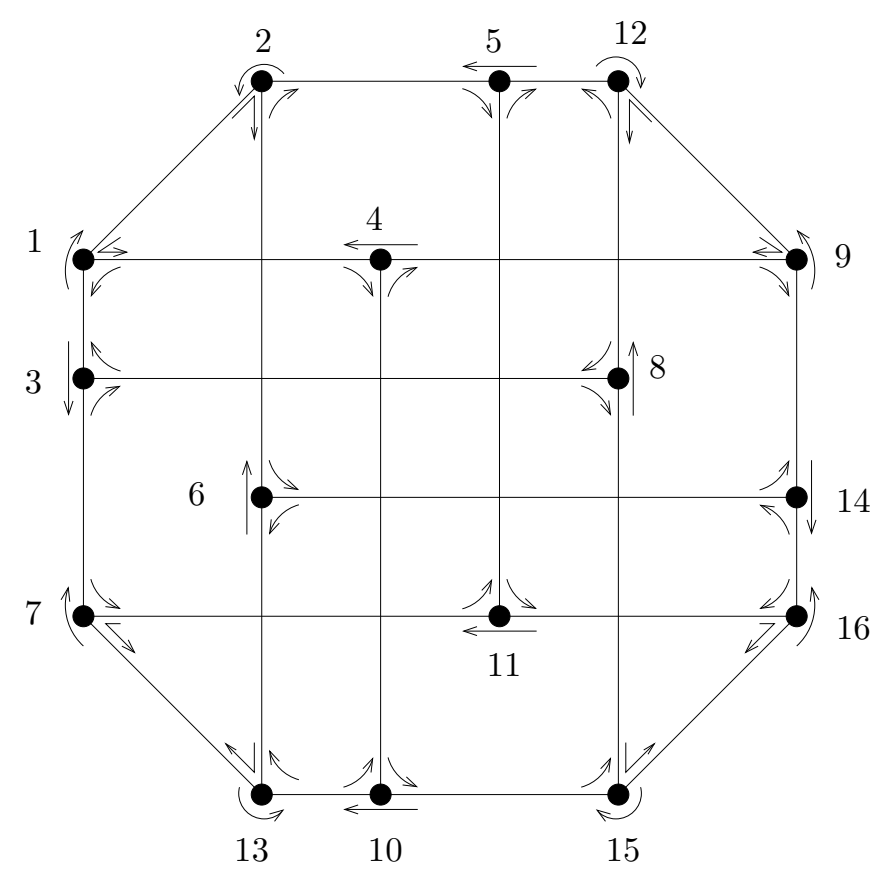

Figure 6: Graph $G_{4060}^{0}$ with orientations that give six 8-cycles.

for the first vertex and thus the labelling for the three edges adjacent to it: 15 triangles to choose from, 3 orientations for each one, see Table 1 for the triangles. Let us pick $\left(x_{1}, x_{5}, x_{2}\right)$ as in Figure 7 Now, for vertex 2, we have two choices: $\left(x_{1}, x_{6}, x_{4}\right)$ or $\left(x_{1}, x_{13}, x_{3}\right)$. Let us take the first one. For vertex 3 can use either $\left(x_{5}, x_{9}, x_{10}\right)$ or $\left(x_{5}, x_{13}, x_{9}\right)$. If we chose the first one, for vertex 5 we would need a triplet with $x_{9}$ followed by $x_{4}$. If we chose the latter one, we would need a triplet with $x_{13}$ followed by $x_{6}$. However, there are no such triangles in 
$T_{21}$, see Table 1 So, our choice for the vertex 2 leads us nowhere. We had also another choice for vertex 2 , namely $\left(x_{1}, x_{13}, x_{3}\right)$. But, again, choosing either of our possibilities $\left(x_{5}, x_{9}, x_{10}\right)$ or $\left(x_{5}, x_{13}, x_{9}\right)$ for vertex 3 makes it impossible to get a triangle for vertex 5 . Thus, the initial choice for the first vertex does not lead to any colouring of the graph.

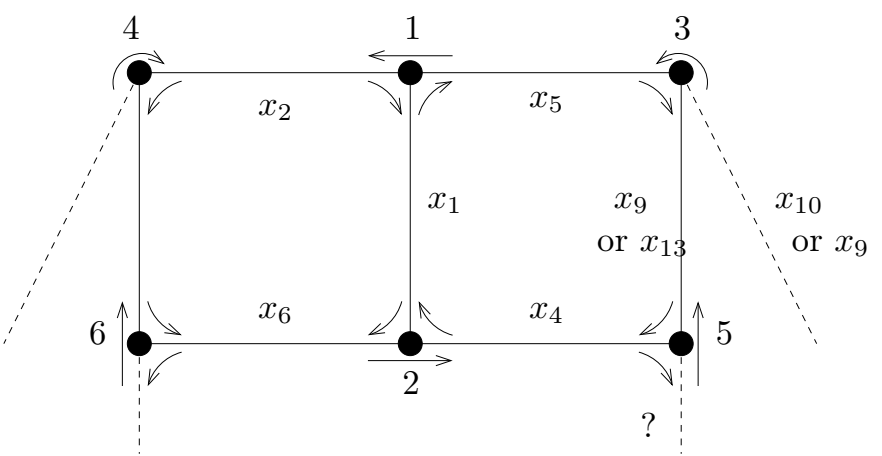

Figure 7: Colouring vertex 1 in the graph $G_{3621}^{0}$ with $\left(x_{1}, x_{5}, x_{2}\right)$, 2 with $\left(x_{1}, x_{6}, x_{4}\right)$ and 3 with either $\left(x_{5}, x_{9}, x_{10}\right)$ or $\left(x_{5}, x_{13}, x_{9}\right)$ leaves no possibility to colour vertex 5 .

With the computer program we try out all possible choices: we try out all 23 sets of triangles for all five candidates for dual graphs, for each of them all ways of picking the six octagons. In each case we try out all 45 choices for the labels around the first vertex. As a result, we find colourings for the graph $G_{3345}^{0}$ by the triangles in the presentations $T_{1}$ and $T_{2}$ listed in [9], but no colouring with any subset of the triangles in any of the other presentations $T_{3}-T_{23}$.

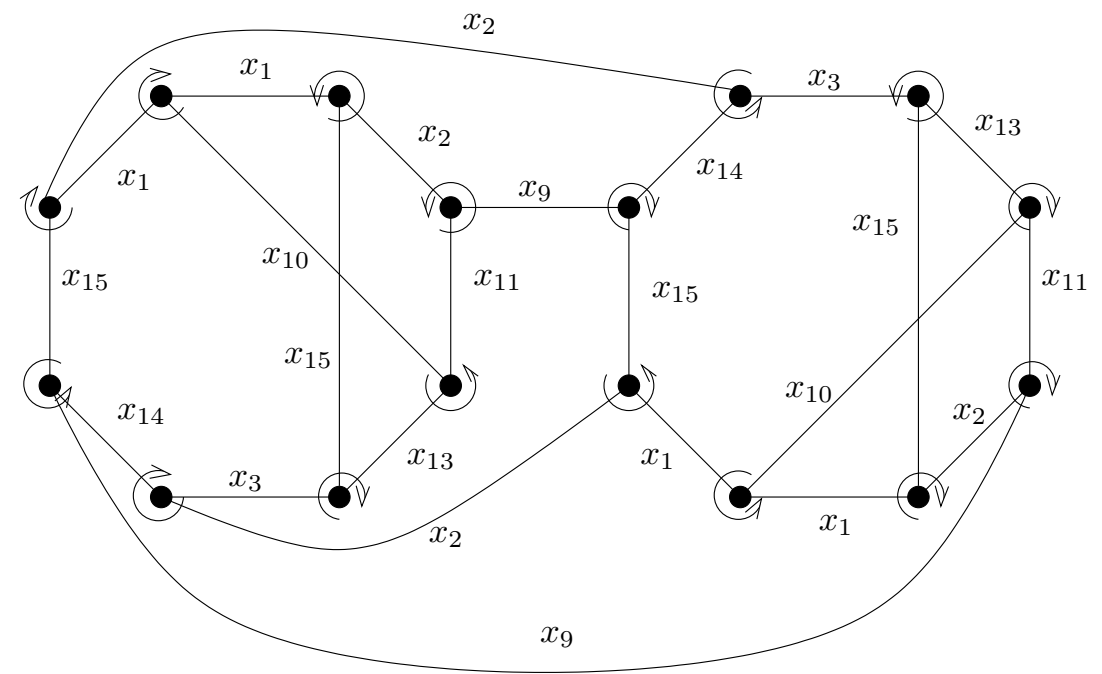

Figure 8: Colouring of the graph $G_{3345}^{0}$ with triangles from $T_{1}$.

A colouring of the graph $G_{3345}^{0}$ with triangles from $T_{1}$ is presented in Figure 8. Thus these 16 triangles, namely $\left(x_{1}, x_{1}, x_{10}\right)$ at vertices 1 and 16 of the 
graph $G_{3345}^{0}$ (see Table 1 for the triangles and Figure 2 for the labelling of the vertices), $\left(x_{1}, x_{15}, x_{2}\right)$ at vertices $2,4,13$ and $15,\left(x_{2}, x_{11}, x_{9}\right)$ at vertices 5 and $12,\left(x_{2}, x_{14}, x_{3}\right)$ at vertices 7 and $10,\left(x_{3}, x_{15}, x_{13}\right)$ at vertices 6 and 11 , $\left(x_{9}, x_{14}, x_{15}\right)$ at vertices 8 and 9 and $\left(x_{10}, x_{13}, x_{11}\right)$ at vertices 3 and 14 , give a surface of genus 2 . Since these 7 triangles used in the colouring are among the 15 triangles of the presentation $T_{2}$ as well, see [9], the same periodic plane exists in $T_{2}$, too. For the other four candidates for dual graphs no colourings can be found.

Thus in the buildings defined by the presentations $T_{1}$ and $T_{2}$ there are periodic planes of genus 2 and therefore surface subgroups of the same genus. However, when considering the possible dual graphs that do not have multiple edges, there are no apartments invariant by genus 2 surface group actions associated to the other 21 triangular presentations.

\subsection{Dual graphs with double edges}

Let us then consider the surfaces where some of the triangles are glued together by two sides. This means that in the dual graph there are two edges between some two vertices. There cannot however be adjacent double edges, that would give degree 4 to the vertex between them. Also seven double edges altogether would make the existence of cycles of length 8 impossible. Thus, we will generate and check all possible dual graphs with six or less double edges.

We generate the possible dual graphs with double edges as follows: For the dual graphs with $n$ double edges we first generate with nauty [10] the connected, bipartite graphs with 16 vertices and $24-n$ edges, with vertices of degree two and three. Then we check whether the vertices of degree two in a graph are pairwise adjacent. If they are, we double these edges to obtain a 3-valent graph with $n$ double edges. See Table 4 for the numbers of graphs obtained.

\begin{tabular}{|c|c|c|}
\hline Double edges & Graphs & Possible dual graphs \\
\hline 0 & 38 & $G_{3345}^{0}, G_{3538}^{0}, G_{3621}^{0}, G_{4002}^{0}, G_{4060}^{0}$ \\
1 & 86 & $G_{61}^{1}, G_{84}^{1}$ \\
2 & 145 & $G_{20}^{2}, G_{25}^{2}, G_{78}^{2}, G_{84}^{2}$ \\
3 & 132 & $G_{112}^{3}$ \\
4 & 75 & - \\
5 & 21 & - \\
6 & 1 & - \\
\hline
\end{tabular}

Table 4: The amounts of graphs with different numbers of double edges

After generating the graphs we run the same depth first search as earlier to see, whether the graphs consist of six cycles of length eight. We obtain two possible dual graphs with one double edge, nine with two double edges, four with three and four with four double edges. With more double edges suitable graphs do not exist.

When studying these graphs further, we see that in fact not even all of these graphs are possible dual graphs. Namely, the 8-cycles in the dual graphs arise from orientations given to the vertices. Thus, if we have a double edge between two vertices, there is only two possibilities how a cycle can go through the ver- 
tices. When the graph is drawn on a plane, either both the vertices are oriented to the same direction as in Figure 9a, or, they have opposite orientations, as in Figure 9b. In the first case a cycle coming in from vertex $C$ towards vertex $A$ would continue to vertex $B$ and $D$ and then further. Similarly for a path coming from $D$ towards $B$. With these two paths the edges $A C$ and $B D$ are travelled twice, but the edges between $A$ and $B$ are not. Thus, the vertices joined by a double edge must have opposite orientations to allow all edges to be travelled twice.

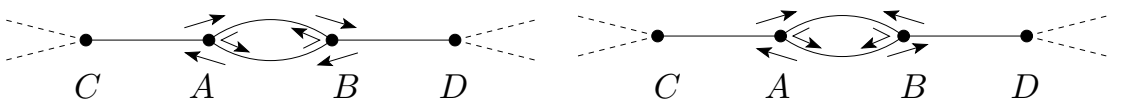

(a) Vertices with same orientation

(b) Vertices with opposite orientations

Figure 9: Possible orientations of vertices joined by a double edge

Now, when the vertices joined by a double edge have opposite orientations, as in Figure $9 \mathrm{~b}$, we immediately have some additional information about the 8-cycles. Namely, a path from $C$ towards $A$ continues to $B$ and then back to $A$. Thus, to get a cycle, this paths needs to continue with a cycle of four edges from $C$. Similarly for a path from $D$ towards $B$. In half of the graphs that are found to have six 8-cycles with the depth first search there are no such cycles of length four available, and thus they are not suitable for dual graphs. Let us call the graphs we have left by $G_{n}^{d}$, where $d$ is the number of double edges, and $n$ is the number of the graph in the list of graphs generated by nauty. Thus we have the two graphs with one double edge, $G_{61}^{1}$ and $G_{84}^{1}$, four graphs with two double edges, $G_{20}^{2}, G_{25}^{2}, G_{78}^{2}$ and $G_{84}^{2}$, and one graph $G_{112}^{3}$ with three double edges, see Table 4. The graphs are presented in Figures 10, 11] and [12.

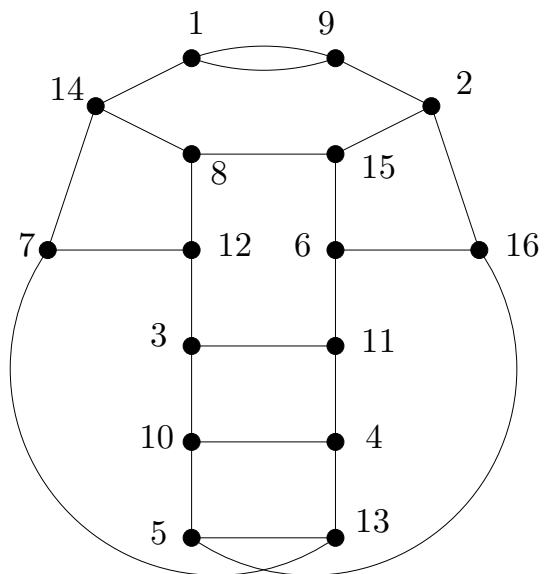

(a) Graph $G_{61}^{1}$

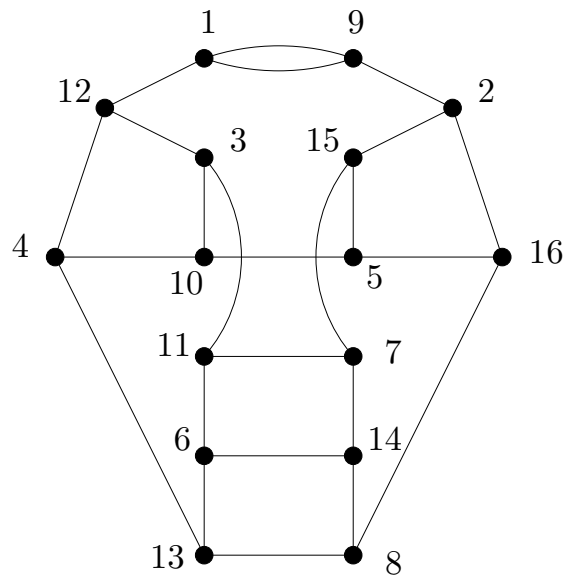

(b) Graph $G_{84}^{1}$

Figure 10: Graphs with one double edge

All of these seven graphs have several possible ways to pick the set of six 8 -cycles. We check by computer for each graph all $2^{16}$ possibilities to orient the vertices in order to see which orientations produce a set of six 8-cycles. As a result we have 8 different sets of six 8-cycles for $G_{61}^{1}, 24$ for $G_{84}^{1}, 64$ for $G_{20}^{2}$ and 


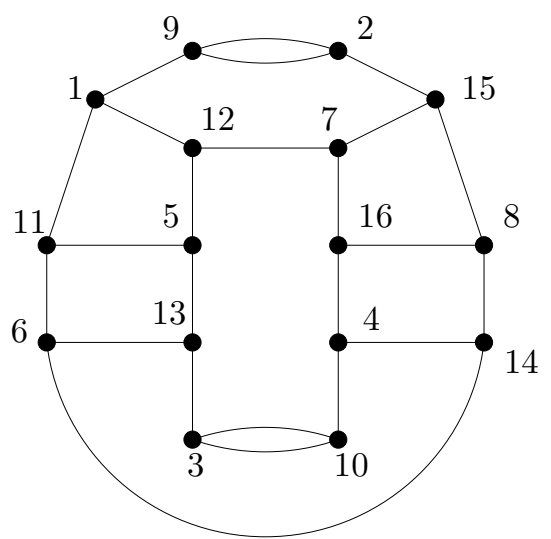

(a) Graph $G_{20}^{2}$

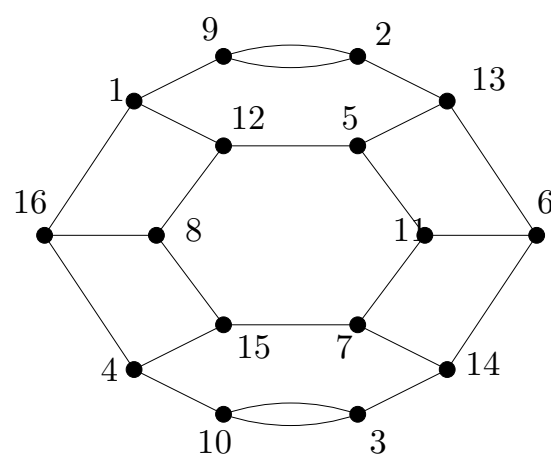

(c) Graph $G_{78}^{2}$

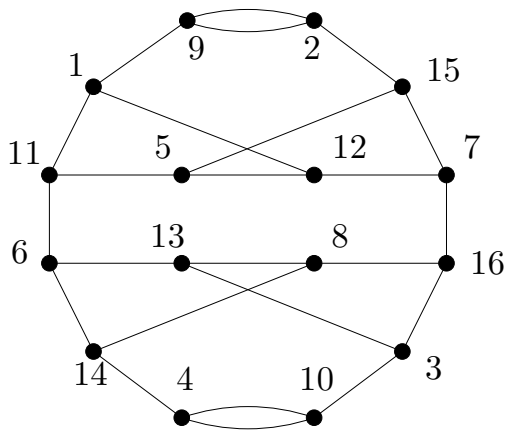

(b) Graph $G_{25}^{2}$

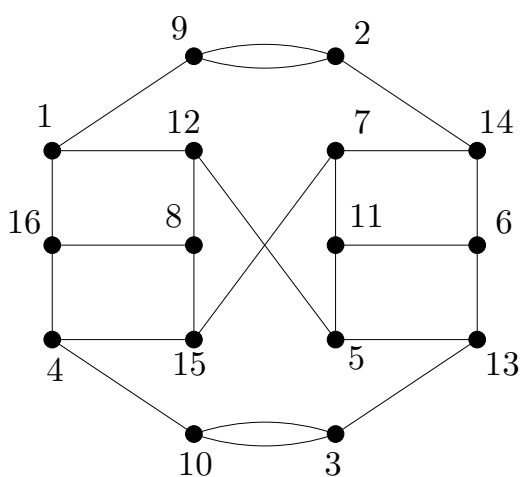

(d) Graph $G_{84}^{2}$

Figure 11: Graphs with two double edges

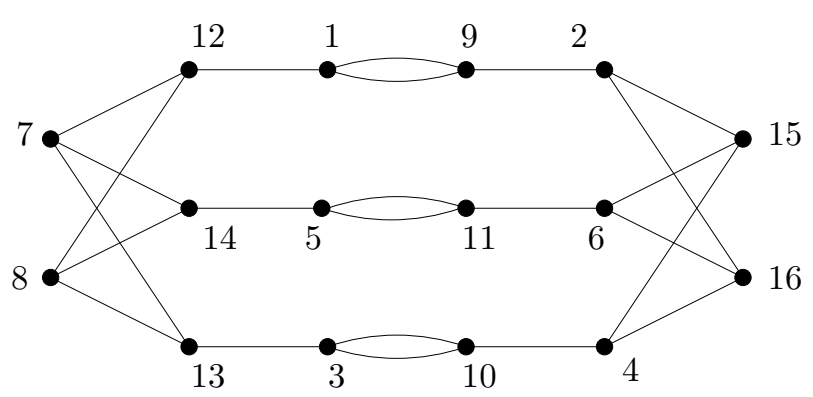

Figure 12: Graph $G 3_{112}$ with three double edges.

$G_{25}^{2}, 32$ for $G_{78}^{2}$ and $G_{84}^{2}$, and 1024 for the graph $G_{112}^{3}$.

Then we search for colourings of the vertices of these seven multigraphs with triangles from the 23 groups in [9], giving to the three edges meeting at a vertex labels from the three sides of one of the triangles. The search is otherwise similar to earlier, but this time we first color the graphs without paying attention to orientations of the vertices and triangles. Only after receiving a candidate for possible coloring we then check, whether the coloring corresponds to one of the 
orientations at the vertices that gives six 8-cycles in the graph.

We obtain colourings for the graphs $G_{78}^{2}, G_{84}^{2}$ and $G_{112}^{3}$, the other four multigraphs do no admit any colourings. The graphs $G_{78}^{2}$ and $G_{84}^{2}$ can be coloured with the triangles from the group $T_{18}$ in $\left[9\right.$. The graph $G_{112}^{3}$ can be coloured with triangles from the groups $T_{1}, T_{7}$ or $T_{9}$. We already knew that for $T_{1}$ a periodic apartment exists in the corresponding building. However, this colouring of $G_{112}^{3}$ by $T_{1}$ uses different triangles than the colouring obtained for $G_{3345}^{0}$, and this periodic apartment is not found in the building corresponding to $T_{2}$. The new cases of periodic apartments with dual graph $G_{112}^{3}$ are in the buildings given by $T_{7}$ and $T_{9}$. The obtained colourings of $G_{84}^{2}$ by $T_{18}$ and of $G_{112}^{3}$ by $T_{7}$ and $T_{9}$ are presented in the Table 5 on line $i$ is the triangle that colours vertex $i$ of the corresponding graph, when the vertices are numbered as in the Figures 11] and 12, The labels on the sides of the cyclic triangle are given in the order matching to the ascending order of the labels of the vertices adjacent to $i$.

\begin{tabular}{|l|l|l|}
\hline Colouring with $T_{7}$ & Colouring with $T_{9}$ & Colouring with $T_{18}$ \\
\hline$\left(x_{7}, x_{3}, x_{4}\right)$ & $\left(x_{14}, x_{14}, x_{4}\right)$ & $\left(x_{15}, x_{13}, x_{5}\right)$ \\
$\left(x_{6}, x_{9}, x_{11}\right)$ & $\left(x_{4}, x_{8}, x_{5}\right)$ & $\left(x_{1}, x_{1}, x_{15}\right)$ \\
$\left(x_{7}, x_{3}, x_{6}\right)$ & $\left(x_{15}, x_{9}, x_{6}\right)$ & $\left(x_{1}, x_{1}, x_{15}\right)$ \\
$\left(x_{4}, x_{15}, x_{13}\right)$ & $\left(x_{13}, x_{3}, x_{10}\right)$ & $\left(x_{15}, x_{10}, x_{12}\right)$ \\
$\left(x_{12}, x_{12}, x_{3}\right)$ & $\left(x_{15}, x_{9}, x_{13}\right)$ & $\left(x_{7}, x_{11}, x_{10}\right)$ \\
$\left(x_{3}, x_{7}, x_{6}\right)$ & $\left(x_{6}, x_{7}, x_{12}\right)$ & $\left(x_{6}, x_{12}, x_{5}\right)$ \\
$\left(x_{13}, x_{11}, x_{6}\right)$ & $\left(x_{8}, x_{7}, x_{3}\right)$ & $\left(x_{9}, x_{13}, x_{11}\right)$ \\
$\left(x_{15}, x_{9}, x_{7}\right)$ & $\left(x_{5}, x_{12}, x_{10}\right)$ & $\left(x_{9}, x_{7}, x_{6}\right)$ \\
$\left(x_{7}, x_{3}, x_{6}\right)$ & $\left(x_{14}, x_{14}, x_{4}\right)$ & $\left(x_{15}, x_{1}, x_{1}\right)$ \\
$\left(x_{7}, x_{3}, x_{4}\right)$ & $\left(x_{15}, x_{9}, x_{13}\right)$ & $\left(x_{1}, x_{1}, x_{15}\right)$ \\
$\left(x_{12}, x_{12}, x_{3}\right)$ & $\left(x_{15}, x_{9}, x_{6}\right)$ & $\left(x_{7}, x_{6}, x_{9}\right)$ \\
$\left(x_{4}, x_{13}, x_{15}\right)$ & $\left(x_{4}, x_{8}, x_{5}\right)$ & $\left(x_{13}, x_{11}, x_{9}\right)$ \\
$\left(x_{6}, x_{11}, x_{9}\right)$ & $\left(x_{6}, x_{7}, x_{12}\right)$ & $\left(x_{15}, x_{10}, x_{12}\right)$ \\
$\left(x_{3}, x_{6}, x_{7}\right)$ & $\left(x_{13}, x_{3}, x_{10}\right)$ & $\left(x_{15}, x_{5}, x_{13}\right)$ \\
$\left(x_{9}, x_{15}, x_{7}\right)$ & $\left(x_{8}, x_{3}, x_{7}\right)$ & $\left(x_{10}, x_{11}, x_{7}\right)$ \\
$\left(x_{11}, x_{13}, x_{6}\right)$ & $\left(x_{5}, x_{10}, x_{12}\right)$ & $\left(x_{5}, x_{12}, x_{6}\right)$ \\
\hline
\end{tabular}

Table 5: Colours for the vertices of $G_{112}^{3}$ from presentations $T_{7}$ and $T_{9}$ and for the vertices of $G_{84}^{2}$ from $T_{18}$ in [9].

We have now went through all possible colourings for all possible dual graphs both with and without multiple edges. As a result we found periodic apartments of genus 2 and therefore surface subgroups in the buildings given by $T_{1}, T_{2}, T_{7}$, $T_{9}$ and $T_{18}$ in [9]. For the other 18 cases presented in [9] no periodic apartment invariant under a genus 2 action exists. This ends the proof of Theorem 2.1

Remark 2.2. From (11) we note immediately that surfaces of genus 0 or 1 are impossible.

Remark 2.3. The existence of periodic apartments of genus 3 could in theory be checked the same way. For them the possible dual graph would have to be bipartite trivalent graphs with 32 vertices, 48 edges and 12 cycles of length 8. However, since there are already 18941522184590 trivalent graphs with 32 ver- 
tices without double edges [7], the calculation time to find the possible graphs and to search colourings with the current algorithms would be too long.

\section{Acknowledgements}

The authors would like to thank Tim Steger for useful discussions. This article was finalized in July 2015 when the authors were invited to work at Max Planck Institute for Mathematics in Bonn. We wish to thank MPIM for their hospitality. We also acknowledge the support of the EPSRC grant EP/K016687/1.

\section{References}

[1] W. Ballmann and M. Brin, Orbihedra of nonpositive curvature, Inst. Hautes Études Sci. Publ. Math. No. 82 (1995), 169-209.

[2] D. Calegari and A. Walker, Random groups contain surface subgroups, to appear in J. Amer. Math. Soc. Preprint available on the arXiv:1304.2188

[3] L. Carbone, R. Kangaslampi, and A. Vdovina, Groups acting simply transitively on vertex sets of hyperbolic triangular buildings, LMS J. Comput. Math. 15 (2012), 101-112.

[4] J. Crisp, M. Sageev, and M. Sapir, Surface subgroups of right-angled Artin groups, Internat. J. Algebra Comput. 18 (2008), no. 3, 443-491.

[5] D. Futer and A. Thomas, Surface quotients of hyperbolic buildings., Int. Math. Res. Not. 2012, no. 2, 437-477.

[6] C.McA. Gordon, D.D. Long, and A.W. Reid, Surface subgroups of Coxeter and Artin groups, J. Pure Appl. Algebra 189 (2004), 135-148.

[7] http://staffhome.ecm.uwa.edu.au/ 00013890/remote/cubics/

[8] A. Hatcher, Algebraic Topology, Cambridge University Press, 2002.

[9] R. Kangaslampi and A. Vdovina, Cocompact actions on hyperbolic buildings, Internat. J. Algebra Comput. 20 (2010), no. 4, 591-603.

[10] B. D. McKay and A. Piperno, Practical Graph Isomorphism, II, J. Symbolic Computation 60 (2013), 94-112.

[11] J. Stillwell. Classical Topoogy and Combinatorial Group Theory, Graduate Texts in Mathematics, Springer-Verlag, New York, 1980.

[12] A. Thomas, Lattices in hyperbolic buildings, to appear in the volume "Geometry, topology, and dynamics in negative curvature - Proceedings", London Math. Soc., Lecture Notes series. Preprint available on the arXiv:1204.0287

[13] A. Vdovina, Combinatorial structure of some hyperbolic buildings, Math. Z. 241 (2002), no. 3, 471-478.

[14] A. Vdovina, Groups, periodic planes and hyperbolic buildings, J. Group Theory 8 (2005), no. 6, 755-765. 